\title{
Signalling strategies for osteogenic differentiation of human umbilical cord mesenchymal stromal cells for $3 D$ bone tissue engineering
}

\author{
Limin Wang, ${ }^{1}$ Milind Singh, ${ }^{2}$ Lynda F. Bonewald ${ }^{3}$ and Michael S. Detamore ${ }^{4 *}$ \\ ${ }^{1}$ Department of Biomedical Engineering, University of Michigan, Ann Arbor, MI 48109, USA \\ ${ }^{2}$ Department of Bioengineering, Rice University, Houston, TX 77251, USA \\ ${ }^{3}$ Department of Oral Biology, University of Missouri-Kansas City, Kansas City, MO 64108, USA \\ ${ }^{4}$ Department of Chemical and Petroleum Engineering, University of Kansas, Lawrence, KS 66045, USA
}

\begin{abstract}
Human umbilical cord mesenchymal stromal cells (hUCMSCs) have recently shown the capacity to differentiate into multiple cell lineages in all three embryonic germ layers. The osteogenic differentiation of hUCMSCs in monolayer culture has been reported, while the differentiation in three-dimensional biomaterials has not yet been reported for tissue-engineering applications. Thus, the aim of this study was to evaluate the feasibility of using hUCMSCs for bone tissue engineering. hUCMSCs were cultured in poly(L-lactic acid) (PLLA) scaffolds in osteogenic medium (OM) for 3 weeks, after which the scaffolds were exposed to several different media, including the OM, a mineralization medium (MM) and the MM with either 10 or $100 \mathrm{ng} / \mathrm{ml}$ insulin-like growth factor (IGF)-1. The osteogenic differentiation was confirmed by the up-regulation of Runx2 and OCN, calcium quantification and bone histology. Switching from the OM to the MM promoted collagen synthesis and calcium content per cell, while continuing in the OM retained more cells in the constructs and promoted higher osteogenic gene expression. The addition of IGF-1 into the MM had no effect on cell proliferation, differentiation and matrix synthesis. In conclusion, hUCMSCs show significant potential for bone tissue engineering and culturing in the OM throughout the entire period is beneficial for osteogenic differentiation of these cells. Copyright $\odot 2009$ John Wiley \& Sons, Ltd.
\end{abstract}

Received 15 August 2008; Revised 5 February 2009; Accepted 17 March 2009

Keywords umbilical cord stroma; stem cells; osteogenesis; IGF-1

\section{Introduction}

Bone tissue engineering is a promising interdisciplinary field, in which cells can be integrated into biomaterials to provide a substitute for bone grafts. Indeed, human bone marrow mesenchymal stromal cells (hBMSCs) have been translated into clinical trials via a tissue-engineering approach to treat bone defects successfully in a 7 year clinical trial (Quarto et al., 2001; Marcacci et al., 2007). However, there are some known disadvantages associated

*Correspondence to: Michael S. Detamore, University of Kansas, Department of Chemical and Petroleum Engineering, 4132 Learned Hall, $1530 \mathrm{~W} 15$ th Street, Lawrence, KS 66045, USA. E-mail: detamore@ku.edu with hBMSCs, such as the relative number of hBMSCs in the marrow, a limited proliferation ability and inferior differentiation potential in aged individuals (Mueller and Glowacki, 2001). Moreover, the invasive and painful harvesting procedure may cause donor site morbidity and complications (Lee et al., 2007).

Recent evidence has shown that human umbilical cord mesenchymal stromal cells (hUCMSCs) are a primitive and multipotent stromal population that shares similar characteristics with hBMSCs (Can and Karahuseyinoglu, 2007; Troyer and Weiss, 2008). hUCMSCs are isolated from the Wharton's jelly of umbilical cords, a tissue routinely discarded after delivery. They are nonhaematopoietic cells that can adhere to plastic surfaces for in vitro expansion and have many surface markers 
that are identical to those of hBMSCs, such as CD73, CD90, and CD105 (Wang et al., 2004; Sarugaser et al., 2005; Lu et al., 2006; Fu et al., 2006; Karahuseyinoglu et al., 2007; Campard et al., 2008). They are multipotent, differentiating along a variety of cell lineages in all germ layers, such as chondrocytes, osteoblasts, adipocytes, myocytes, neurons and hepatocytes (Wang et al., 2004; Sarugaser et al., 2005; Lu et al., 2006; Fu et al., 2006; Karahuseyinoglu et al., 2007; Campard et al., 2008). Apart from the similarity to hBMSCs, hUCMSCs have a number of particular advantages over hBMSCs, such as extensive availability and supply, no donor site morbidity, fast proliferation and great in vitro expansion ability, similar to other fetal stem cells. All of these features render hUCMSCs attractive for tissue engineering as a mesenchymal stromal cell (MSC) source.

hUCMSCs have demonstrated their osteogenic differentiation in two-dimensional (2D) monolayer culture after exposure to chemical signals containing dexamethasone and $\beta$-glycerophosphate (Wang et al., 2004; Sarugaser et al., 2005; Lu et al., 2006; Karahuseyinoglu et al., 2007; Wu et al., 2007; Baksh et al., 2007) and osteoinductive biomaterials such as demineralized bone matrix (Honsawek et al., 2006). However, most studies regarding osteogenic differentiation of hUCMSCs are reported briefly in a qualitative way, with histological stains such as Alizarin red S and von Kossa (Wang et al., 2004; Sarugaser et al., 2005; Lu et al., 2006; Karahuseyinoglu et al., 2007; Wu et al., 2007; Baksh et al., 2007). In addition, osteogenic differentiation of hUCMSCs in three-dimensional (3D) biomaterials in vitro is scarcely explored in the literature, with only one study using an osteoinductive material, polycaprolactone tricalcium phosphate (Zhang et al., 2008). In our group, we have previously shown that osteogenic differentiation can be achieved in polyglycolic acid (PGA) scaffolds, although the rapid degradation rate of PGA led to a loss of cells and construct integrity (unpublished data). Like PGA, poly(Llactic acid) (PLLA) has been extensively investigated in tissue-engineering applications, with good biodegradability, biocompatibility and mechanical properties, but is more slowly degraded. This slower degradation is more desirable in vitro, although the ideal degradation rate in vivo may lie somewhere between PGA and PLLA degradation rates. In a phosphate-buffered saline (PBS) solution at $37^{\circ} \mathrm{C}$, quenched PGA demonstrated a rapid decrease in mass after 10 days (Hurrell and Cameron, 2001), while in another study (Yuan et al., 2002) PLLA meshes had almost no loss of mass after 35 weeks in the same buffer. Therefore, non-woven PLLA meshes were used in the current study as support for cell growth and bone formation during the in vitro osteogenesis of hUCMSCs.

The majority of bone tissue engineering studies using BMSCs have been conducted with an osteogenic medium (OM) throughout the entire culture period (up to 6 weeks) (Yoshimoto et al., 2003; Meinel et al., 2004), although osteogenic differentiation of BMSCs can be accomplished in 2-3 weeks, with extensive mineralization (Jaiswal et al., 1997; Pittenger et al., 1999; Sumanasinghe et al.,
2008). However, the differentiation factors, including dexamethasone and 1 $\alpha, 25$-dihydroxyvitamin D3, may inhibit cell proliferation (Walsh et al., 2001; Atmani et al., 2003; Chen, 2004; Kim et al., 2006) for both BMSCs and osteoblasts. Moreover, it is unknown how the OM affects differentiated MSCs. Thus, in the current study, we cultured hUCMSCs with the OM in slowly degrading PLLA scaffolds for 3 weeks, and then removed osteogenic components (dexamethasone and vitamin $\mathrm{D}_{3}$ ) to expose pre-differentiated hUCMSCs to a mineralization medium (MM) and an insulin-like growth factor (IGF)-1containing MM for an additional 3 weeks. The negative and positive controls were none or continued exposure to osteogenic components, respectively. The goal of this study was to examine in vitro osteogenesis with both qualitative and quantitative data and to determine the effects of the medium shift on osteogenically induced hUCMSCs.

\section{Materials and methods}

\subsection{Isolation and culture of hUCMSCs}

IRB approval was obtained for human umbilical cord collection and hUCMSC isolation from KU-Lawrence (No. 15 402) and the KU Medical Center (No. 10951). In this study, two human umbilical cords (both female, 15 and $23 \mathrm{~cm}$ long) were first cut into $3-5 \mathrm{~cm}$ segments, from which vessels were then removed. Cord segments were cut into $1-2 \mathrm{~mm}^{3}$ pieces and incubated at $37^{\circ} \mathrm{C}$ in $0.75 \mathrm{mg} / \mathrm{ml}$ type II collagenase $(298 \mathrm{U} / \mathrm{mg}$; Worthington Biochemical; Lakewood, NJ, USA). A $5 \mathrm{~h}$ incubation yielded a homogeneous gelatinous solution, which was then diluted $(1: 8)$ in sterile phosphate-buffered saline (PBS). hUCMSCs were obtained by centrifugation and then resuspended in an expansion medium containing low-glucose Dulbecco's modified Eagle's medium (DMEMLG; Invitrogen, Carlsbad, CA, USA), 10\% MSC-qualified fetal bovine serum (FBS; Stem Cell Technologies, Vancouver, BC, Canada) and 1\% penicillin/streptomycin (PS; Invitrogen). Cells were plated in cell culture flasks at 8000 cells $/ \mathrm{cm}^{2}$, expanded to $80-90 \%$ confluence (recorded as P0 cells) and detached by $1 \times$ trypsin (Invitrogen). Following a previously described procedure (Wang et al., 2008a), P0 cells were cryogenically stored in liquid nitrogen at $-196^{\circ} \mathrm{C}$ for future use.

\subsection{Scaffold preparation, cell seeding and differentiation}

hUCMSCs were thawed and expanded in the expansion medium to P4 for cell seeding. Cylinder-shaped scaffolds ( $n=83$ ), $5 \mathrm{~mm}$ in diameter and $2 \mathrm{~mm}$ in thickness, were cut out of non-woven PLLA meshes (>95\% porosity and 45-55\% crystallinity; Biomedical Structures, Warwick, RI, USA). The scaffolds were sterilized in a sterile pouch, using ethylene oxide, and then placed in a fume hood. 
Before seeding, scaffolds were wetted with sterile-filtered ethanol followed by two PBS washes, and then immersed for 1 day in the expansion medium. P4 hUCMSCs were resuspended in the expansion medium and then seeded at $25 \times 10^{6}$ cells $/ \mathrm{ml}$ of scaffold into PLLA scaffolds, using an orbital shaker at $150 \mathrm{rpm}$ according to a previously described protocol (Wang et al., 2008b). The expansion medium was referred as to the control medium (CM) over the following culture period. After a 2 day seeding period (week 0 ), some of the scaffolds were continuously cultured in the CM and the remaining ones were cultured in the $\mathrm{OM}$ by replacing the $\mathrm{CM}$ with $1.5 \mathrm{ml} \mathrm{OM}$, consisting of the CM supplemented by $100 \mathrm{~nm}$ dexamethasone (DEX; Sigma, St. Louis, MO, USA), $5 \mathrm{~mm} \beta$-glycerophosphate ( $\beta$-GP; Sigma), 10 nM $1 \alpha, 25$-dihydroxyvitamin D3 (VD3; Biomol International, Plymouth Meeting, PA, USA) and $50 \mu \mathrm{g} / \mathrm{ml}$ ascorbic acid 2-phosphate (AA2P; Sigma). After 3 weeks there were five different culture conditions: (a) continue culture in the CM; (b) continue culture in the OM; (c) change culture from the OM to the MM, which consisted of the OM without DEX and VD3; (d) change culture from the OM to the MM with $10 \mathrm{ng} / \mathrm{ml}$ IGF-1 (I10); or (e) change culture from the OM to the MM with $100 \mathrm{ng} / \mathrm{ml} \mathrm{IGF-1} \mathrm{(I100).} 1 \mathrm{ml}$ medium was changed every other day, except for the complete replacement at weeks 0 and 3.

\subsection{Biochemical assays}

Constructs $(n=4)$ were homogenized in $1.2 \mathrm{ml}$ papain solution $(120 \mu \mathrm{g} / \mathrm{ml})$ and then incubated at $60^{\circ} \mathrm{C}$ overnight. A PicoGreen kit (Invitrogen) was used to determine DNA contents, according to the manufacturer's protocol. A hydroxyproline (HYP) assay was used to obtain HYP content, using a previously described protocol (Wang et al., 2008a). A conversion factor of $8.5 \mathrm{pg}$ DNA/cell was used to convert DNA content to cell number, and a conversion factor of 11.5 can be used to convert HYP mass to collagen mass, based on our preliminary studies.

Calcium content was quantified by an OCPC (orthocresolphthalein complex one) method as described in the literature (ter Brugge and Jansen, 2002). Constructs $(n=4)$ were homogenized and suspended in $1 \mathrm{~N}$ acetic acid (Sigma) overnight. $50 \mu \mathrm{l}$ of this culture sample or a standard solution (0-100 mg/ml $\mathrm{CaCl}_{2}$; Sigma) was added into $250 \mu \mathrm{l}$ of working solution. The working solution contained $0.05 \mathrm{mg} / \mathrm{ml}$ OCPC solution $(1 \mathrm{mg} / \mathrm{ml}$ OCPC, $0.0063 \mathrm{~N} \mathrm{KOH}$ and $0.0031 \mathrm{~N}$ acetic acid; Sigma), $0.74 \mathrm{M}$ ethanolamine/boric acid buffer, $\mathrm{pH} 11$ (Sigma) and 2\% 8-hydroxyquinoline (5 $\mathrm{g}$ in $100 \mathrm{ml}$ 95\% ethanol; Sigma). This mixture was incubated at room temperature for $10 \mathrm{~min}$ and then read at $575 \mathrm{~nm}$.

\subsection{Histology}

Frozen sections (10 $\mu \mathrm{m}, n=2)$ were used for histological analysis. Alizarin red $\mathrm{S}$ and von Kossa staining were used to visualize the mineralization. Sections were fixed in $10 \%$ phosphate-buffered formalin for $20 \mathrm{~min}$ and then rinsed in ultrapure water before staining. For Alizarin red S staining, sections were incubated with 2\% Alizarin red S reagent, $\mathrm{pH}$ 4.1-4.3 (Sigma) for $5 \mathrm{~min}$. For von Kossa staining, sections were incubated with $1 \%$ silver nitrate solution (Sigma) under ultraviolet light for $20 \mathrm{~min}$ and then with $5 \%$ sodium thiosulphate (Sigma) for $5 \mathrm{~min}$ to remove unreacted silver. Finally, all sections were rinsed in ultrapure water and dehydrated through graded alcohols (95\% and 100\%, twice each).

\subsection{RNA isolation and real-time RT-PCR}

Total RNA was extracted from cells using $1 \mathrm{ml}$ Trizol reagent (Invitrogen), following the manufacturer's instructions $(n=4)$. Total RNA concentration and purity were determined on a spectrophotometer (Nanodrop; Wilmington, DE, USA). The mRNA samples were then converted to cDNA using a high-capacity cDNA archive kit (Applied Biosystems, Foster City, CA, USA), following the supplier's procedure. TaqMan gene expression assay kits (Applied Biosystems) were used for transcript levels of type I collagen (CI), runt-related transcription factor 2 (Runx2), and osteocalcin (OCN), using a real-time reverse transcriptase polymerase chain reaction (RT-PCR) in an Applied Biosystems 7500 system. TaqMan gene expression assay kits (Applied Biosystems) were Hs00164004_m1 for CI, Hs00231692_m1for Runx2, Hs01587813_g1 for OCN and Hs99999905_m1 for glyceraldehyde 3-phosphate dehydrogenase (GAPDH). A $2^{-\Delta \Delta \mathrm{Ct}}$ method was used to evaluate relative the mRNA expression level for each target gene (Livak and Schmittgen, 2001). Briefly, $\triangle \mathrm{Ct}$ values were obtained by the difference between the Ct values of target genes and the GAPDH gene. They were then normalized by subtracting the $\Delta \mathrm{Ct}$ value of the calibrator sample, their respective $\mathrm{Ct}$ values in the CM groups, to obtain $\Delta \Delta \mathrm{Ct}$ values.

\subsection{Statistical analysis}

All data were expressed as means \pm one standard deviation (SD) and analysed by one-way ANOVAs, followed by Tukey's Honestly Significant Difference post hoc tests. Statistical significance was determined by a statistical threshold of $p<0.05$.

\section{Results}

\subsection{Cell number}

Over the 6 week culture period, cell number decreased in all groups $(p<0.05)$ compared to week 0 (Figure 1 ). Despite the cell loss throughout the culture period, the OM groups at weeks 3 and 6 retained 1.67 and 1.50 times more cells in the constructs than the CM groups, 


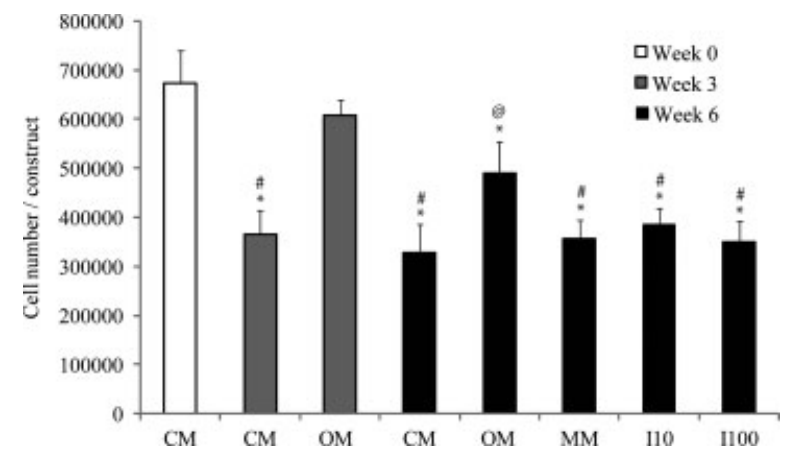

Figure 1. Cell number per construct $(n=4)$. CM, control medium; OM, osteogenic medium; MM, mineralization medium; I10, MM containing $10 \mathrm{ng} / \mathrm{ml}$ IGF-1; I100, MM containing $100 \mathrm{ng} / \mathrm{ml}$ IGF-1. Cell number decreased with time, while the OM group retained more cells than other groups at each time-point. ${ }^{*}$ Statistically significant difference $(p<0.05)$ from week 0 . ${ }^{\#}$ Statistically significant difference $(p<0.05)$ from the OM group at weeks 3 or $6 .{ }^{\circledR}$ Statistically significant difference $(p<0.05)$ in the OM group between weeks 3 and 6. Error bars represent standard deviation (SD). A conversion factor of 8.55 pg DNA/cell was used to convert DNA content to cell number

respectively $(p<0.05)$. During weeks $0-3$, the cell number decreased by $46 \%$ in the CM group $(p<0.05)$, while only decreasing by $10 \%$ in the OM group, without statistical significance. In contrast, during weeks 3-6, the cell number decreased in the CM group only by $10 \%$ without statistical significance, while decreasing by $19 \%$ in the OM group $(p<0.05)$. The medium change at week 3 (from OM to MM, I10, and I100) caused a decrease in cell number $(p<0.05)$ and there were no significant
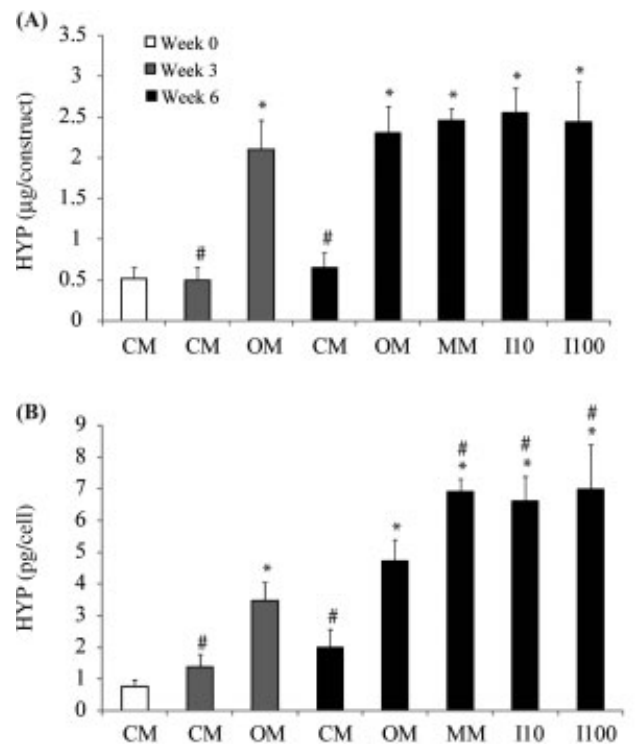

differences in cell number between the MM, I10 and I100 groups.

\subsection{HYP content}

In all groups except the CM group, the HYP content per construct and per cell increased over time, with a substantial leap during weeks $0-3(p<0.05)$ (Figure 2A, $\mathrm{B})$. In the CM groups, the HYP content per construct did not vary over the 6 week culture period and the HYP content per cell had a slight increase, without statistical significance. The OM group had 4.2 and 3.5 times more HYP per construct than the CM group at weeks 3 and 6 , respectively $(p<0.05)$, and had 2.5 and 2.4 times more HYP per cell than the CM group at weeks 3 and 6 , respectively $(p<0.05)$. The shift from OM to MM, I10 or I100 did not affect the total HYP content per construct, while this change did lead to an upregulation of HYP synthesis, given that the HYP content per cell was approximately 50\% greater in the MM, I10 and I100 groups compared to the OM group at week $6(p<0.05)$. Like the cell number, HYP synthesis was not influenced by the addition of IGF-1 into the MM.

\subsection{Calcium content}

There was a considerable increase in calcium content per construct and per cell with time in all groups over the culture period $(p<0.05)$ (Figure $2 \mathrm{C}$ and $2 \mathrm{D})$. Calcium deposition was also observed in the CM group, albeit at
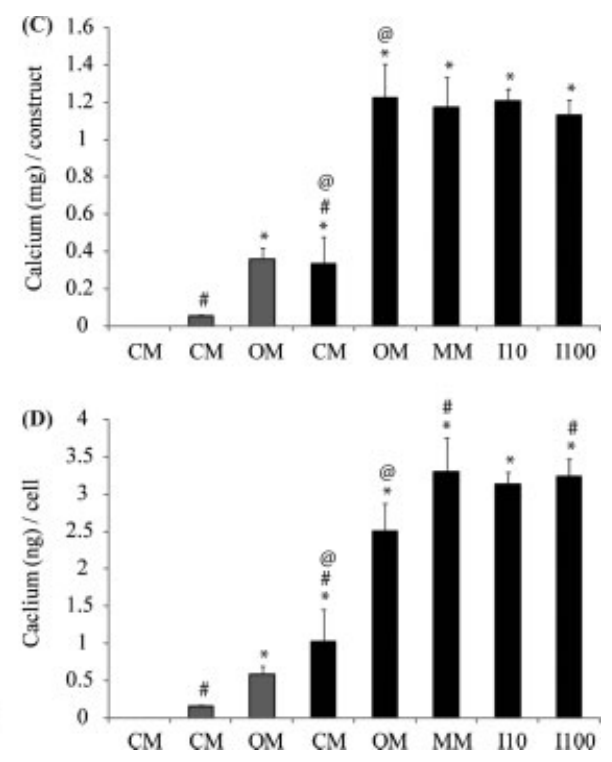

Figure 2. Hydroxyproline (HYP) content per construct (A) and per cell (B) and calcium content per construct (C) and per cell (D) $(n=4)$. CM, control medium; OM, osteogenic medium; MM, mineralization medium; I10, MM containing $10 \mathrm{ng} / \mathrm{ml}$ IGF-1; I100, MM containing $100 \mathrm{ng} / \mathrm{ml}$ IGF-1. (A, B) The OM group had a substantial increase in HYP content per construct and per cell during weeks 0-3 and the CM group had less HYP content than the other groups. The OM group had a comparable HYP content per construct (A) to the MM and IGF groups, while it had less HYP per cell than the MM and IGF groups (B). (C, D) The calcium content per construct and per cell increased over time in all groups and the OM group had more calcium than the CM group. The MM and I100 groups had more calcium per cell than the OM group, while the calcium per construct was comparable among them. "Statistically significant difference $(p<0.05)$ from week 0 . \# Statistically significant difference $(p<0.05)$ from the OM group at weeks 3 or $6 .{ }^{@}$ Statistically significant difference $(p<0.05)$ between weeks 3 and 6. Error bars represent SD 

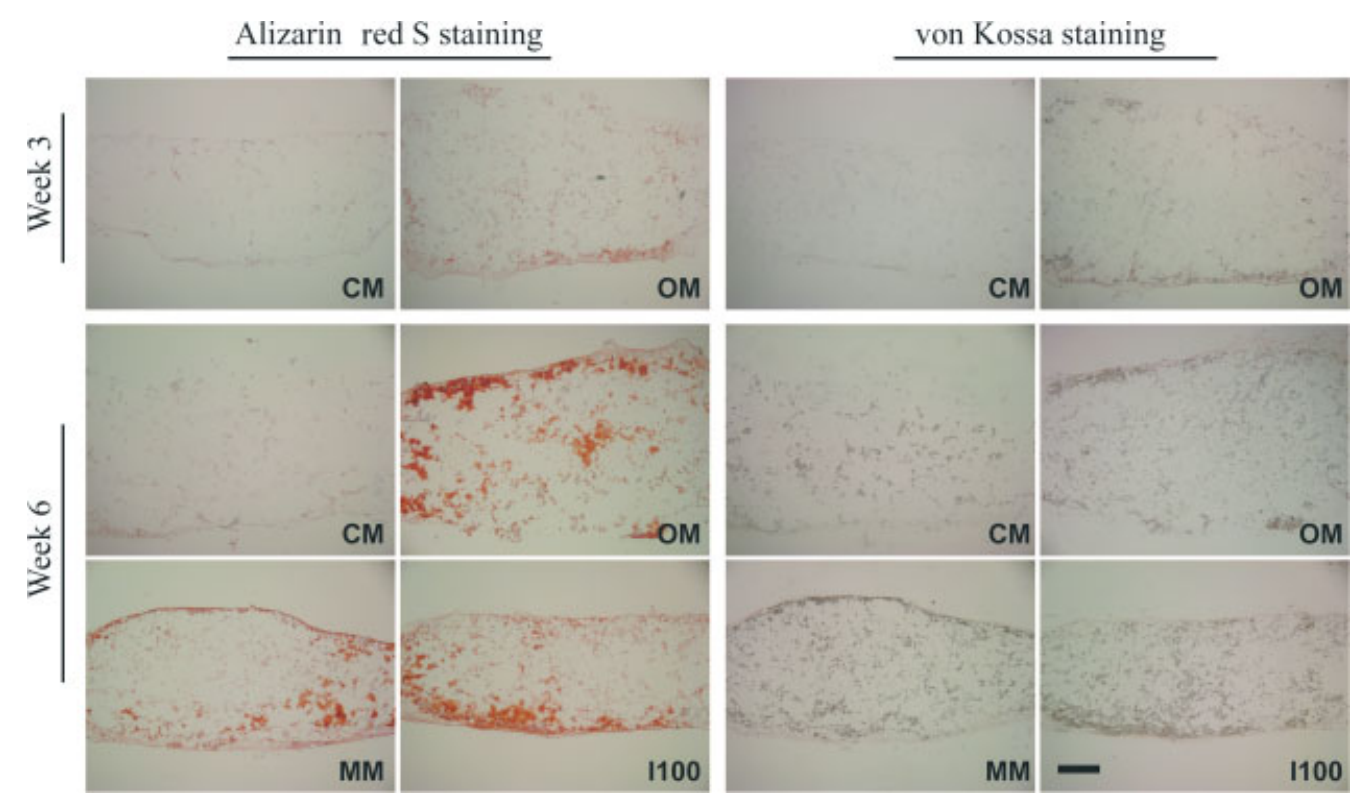

Figure 3. Alizarin red S staining and von Kossa staining at 3 and 6 weeks $(n=2)$. CM, control medium; OM, osteogenic medium; MM, mineralization medium; and I100, MM containing $100 \mathrm{ng} / \mathrm{ml}$ IGF-1. Both types of staining revealed positive mineralization with time. Note that the peripheral area in the constructs had stronger staining than the central area. Scale bar $=500 \mu \mathrm{m}$

a low level. However, the OM group did exhibit more calcium per construct and per cell than the CM group at weeks 3 and $6(p<0.05)$. At week 6 , there was no significant difference in calcium content per construct among the OM, MM, I10 and I100 groups, while the MM and I100 groups had 1.32 and 1.29 times more calcium content per cell than the OM group, respectively $(p<0.05)$. No significant differences were observed in calcium content per cell among the MM, I10 and I100 groups.

\subsection{Histology}

Alizarin red S staining was consistent with von Kossa staining, both of which revealed a positive mineral deposition that increased with time (Figure 3). The CM groups had weaker staining than the OM groups, which was consistent with the calcium quantification. It must be noted that there was a stronger staining at the peripheral area of the constructs than inside the constructs. There were no discernible differences among the OM, MM and I100 groups.

\subsection{Gene expression levels of type I collagen (CI), Runx2 and osteocalcin (OCN)}

Runx2 and OCN gene expression was upregulated in the OM group $(p<0.05)$ (Figure 4$)$. There were no statistically significant differences in $C I$ gene expression among all groups. After a change from the OM, the MM group had a lower gene expression of CI, Runx2 $(p<0.05)$ and $O C N$ when compared to continuing treatment with the OM medium. The addition of IGF-1 into the MM did

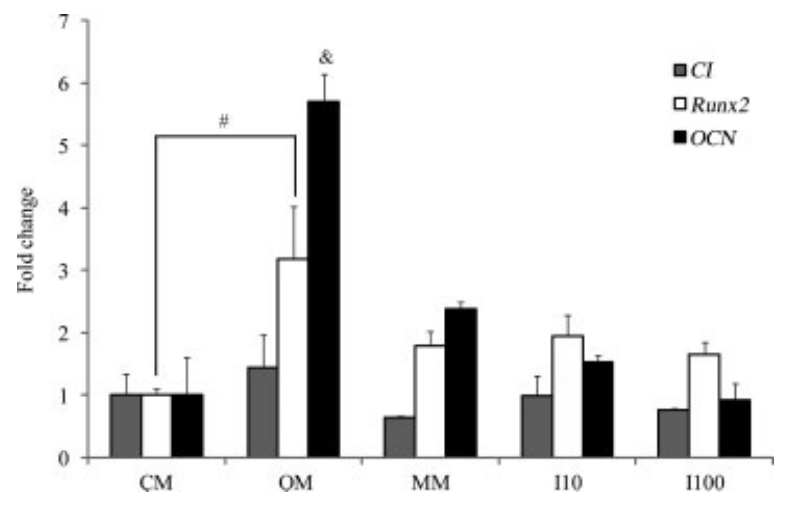

Figure 4. Gene expression (normalized to the $\mathrm{CM}$ group) for type I collagen (CI), runt-related transcription factor 2 (Runx2) and osteocalcin $(O C N)$ at week $6(n=4)$. CM, control medium; OM, osteogenic medium; MM, mineralization medium; I10, MM containing $10 \mathrm{ng} / \mathrm{ml}$ IGF-1; I100, MM containing $100 \mathrm{ng} / \mathrm{ml}$ IGF-1. The OM group had higher Runx2 and OCN mRNA levels than the other groups. \# Statistically significant difference in Runx2 gene expression between the OM and CM groups $(p<0.05) .{ }^{\&}$ Statistically significant difference in $O C N$ gene expression between the OM group and all other groups $(p<0.05)$. Error bars represent SD

not statistically affect the gene expression level of Runx2, OCN or CI.

\section{Discussion and conclusion}

In the field of tissue engineering, it is critical to select a promising cell source that is easy to obtain and provides sufficient cell numbers. hUCMSCs undoubtedly meet these requirements. There is an abundant supply of umbilical cords, with 4.1 million births in the USA alone in 2005 (Martin et al., 2007). After cell isolation, 
hUCMSCs can achieve a 300-fold cell increase in seven passages while maintaining differentiation potential (Karahuseyinoglu et al., 2007). In vivo transplantation of these cells has demonstrated their regenerative effects on brain injuries and retinal disease in animal models, while immunorejection was not observed in these studies (Jomura et al., 2007; Lund et al., 2007). Previous osteogenic differentiation studies with hUCMSCs have been mainly performed in monolayer (2D) (Wang et al., 2004; Sarugaser et al., 2005; Lu et al., 2006; Karahuseyinoglu et al., 2007; Wu et al., 2007; Baksh et al., 2007). In the current study, we investigated the osteogenesis of hUCMSCs in vitro in a 3D biomaterialbased environment, and the effects of different media on osteogenic differentiation, in order to evaluate the use of hUCMSCs for bone tissue engineering.

The Runx2 gene is known to play an essential role in osteoblast differentiation and bone formation (Ducy et al., 1997; Schroeder et al., 2005). The disruption of Runx2 leads to the termination of intramembranous and endochondral ossification, due to the maturational arrest of osteoblasts (Komori et al., 1997). The in vitro osteogenic differentiation of MSCs is accompanied by the upregulation of Runx2 gene expression at the early stage (Tou et al., 2003). The OCN gene is mainly expressed by mature osteoblasts and is responsible for mineralization at the late stage of differentiation. Exposed to the OM, hUCMSCs did show an upregulation of the gene expression of Runx2 and OCN compared to the CM group, indicating osteogenic differentiation of these cells. Compared to the MM, I10 and I100 groups, the $\mathrm{OM}$ is also beneficial for osteogenic differentiation due to the higher Runx2 and OCN gene expression. The positive staining of Alizarin red $\mathrm{S}$ and von Kossa confirmed the presence of mineralization, suggesting that the hUCMSCs were progressing along an osteogenic lineage. In contrast to mineralization, collagen content increased mainly between weeks $0-3$ and was maintained between weeks 3-6. In native normal bone, the nucleation and development of mineral crystals interacts principally with collagen (Landis, 1999). The abundant collagen production may have contributed to the boost of mineralization between weeks 3 and 6 .

The OM also promoted cell proliferation and/or inhibited cell loss compared to other media. This effect was mainly due to the presence of DEX and VD3 in the $\mathrm{OM}$, rather than $\mathrm{AA} 2 \mathrm{P}$ and $\beta$-GP, based on the observation that the MM group had a comparable cell number range with the CM group after switching the medium at week 3. A few studies have demonstrated an inhibitory effect of either DEX or vitamin D3 on cell proliferation (Walsh et al., 2001; Murata et al., 2004; Campbell et al., 1997), although they can induce osteogenic differentiation (Jaiswal et al., 1997; Partridge et al., 1980; Beresford et al., 1980). In monolayer culture, we also observed in a previous study that DEX inhibited hUCMSC proliferation (unpublished data). Thus, the increase in cell number with the OM group in the current study indicated that the combination of DEX and VD3 might have a synergistic effect on hUCMSC proliferation and/or survival. It must be noted that this stimulation decreased with time, and might reach a plateau in cell number similar to the CM group after a longer culture period, which would be of interest for future investigation.

The MM had no impact on the total collagen and calcium content in the constructs, although these had slightly higher collagen and calcium per cell. Moreover, the shift from the OM to the MM led to a decrease in osteogenic gene expression, including CI, Runx2 and OCN. IGF-1 plays an important role as an anabolic agent in bone development at all stages (Linkhart et al., 1996; Ross et al., 1993; Kasukawa et al., 2004) and regulates MSC proliferation and biosynthesis (Jeong et al., 2008; Koch et al., 2005). In the current study, the addition of IGF-1 into the MM had no beneficial effects on cell proliferation, matrix production or gene expression. In the future, the incorporation of IGF-1 at an earlier stage and/or in addition to osteogenic signals, as opposed to in lieu of them, might facilitate the osteogenic differentiation of MSCs (Koch et al., 2005).

In summary, the results demonstrated the osteogenic differentiation of hUCMSCs in PLLA scaffolds, with the upregulation of bone-specific genes and mineral deposition, thus supporting the feasibility of applying hUCMSCs to bone tissue engineering. Therefore, given the numerous advantages over adult stem cells and the successful osteogenesis in 3D biomaterials, hUCMSCs may be a promising alternative for bone tissue engineering. A sideby-side comparison between hUCMSCs and fibroblasts or osteoblasts will be interesting and meaningful to further evaluate the potential of hUCMSCs in bone tissue engineering. In the current study, there was no advantage to removing DEX and VD3 after 3 weeks, as in fact the only group to retain these factors (i.e. the OM group) produced a greater number of cells and expressed the highest level of osteogenic genes. Future strategies may include earlier introduction of IGF-1 and/or extended exposure of DEX and VD3.

\section{Acknowledgements}

This work was supported by the Arthritis Foundation (to MSD), both from the national organization and from the Kansas Chapter, and the USAMRMC (No. 07040004) (to LFB). We would like to thank the staff in the labour and delivery unit at the University of Kansas Medical Center for their assistance with umbilical cord collection. We would also like to thank Nathan Dormer for critical reading of the manuscript.

\section{References}

Atmani H, Chappard D, Basle MF. 2003; Proliferation and differentiation of osteoblasts and adipocytes in rat bone marrow stromal cell cultures: effects of dexamethasone and calcitriol. $J$ Cell Biochem 89: 364-372.

Baksh D, Yao R, Tuan RS. 2007; Comparison of proliferative and multilineage differentiation potential of human mesenchymal stem cells derived from umbilical cord and bone marrow. Stem Cells 25: 1384-1392. 
Beresford JN, Joyner CJ, Devlin C, Triffitt JT. 1994; The effects of dexamethasone and 1,25-dihydroxyvitamin D3 on osteogenic differentiation of human marrow stromal cells in vitro. Arch Oral Biol 39: 941-947.

Campard D, Lysy PA, Najimi M, Sokal EM. 2008; Native umbilical cord matrix stem cells express hepatic markers and differentiate into hepatocyte-like cells. Gastroenterology 134: 833-848.

Campbell MJ, Reddy GS, Koeffler HP. 1997; Vitamin D3 analogs and their 24-oxo metabolites equally inhibit clonal proliferation of a variety of cancer cells but have differing molecular effects. $J$ Cell Biochem 66: 413-425.

Can A, Karahuseyinoglu S. 2007; Concise review: human umbilical cord stroma with regard to the source of fetus-derived stem cells. Stem Cells 25: 2886-2895.

Chen TL. 2004; Inhibition of growth and differentiation of osteoprogenitors in mouse bone marrow stromal cell cultures by increased donor age and glucocorticoid treatment. Bone 35: 83-95.

Ducy P, Zhang R, Geoffroy V, et al. 1997; Osf2/Cbfa1: a transcriptional activator of osteoblast differentiation. Cell 89: 747-754.

Fu YS, Cheng YC, Lin MY, et al. 2006; Conversion of human umbilical cord mesenchymal stem cells in Wharton's jelly to dopaminergic neurons in vitro: potential therapeutic application for Parkinsonism. Stem Cells 24: 115-124.

Honsawek S, Dhitiseith D, Phupong V. 2006; Effects of demineralized bone matrix on proliferation and osteogenic differentiation of mesenchymal stem cells from human umbilical cord. J Med Assoc Thai 89(suppl 3): S189-195.

Hurrell S, Cameron RE. 2001; Polyglycolide: degradation and drug release. Part I: changes in morphology during degradation. J Mater Sci Mater Med 12: 811-816.

Jaiswal N, Haynesworth SE, Caplan AI, Bruder SP. 1997; Osteogenic differentiation of purified, culture-expanded human mesenchymal stem cells in vitro. J Cell Biochem 64: 295-312.

Jeong WK, Park SW, Im GI. 2008; Growth factors reduce the suppression of proliferation and osteogenic differentiation by titanium particles on MSCs. $J$ Biomed Mater Res A 86A: 1137-1144.

Jomura S, Uy M, Mitchell K, et al. 2007; Potential treatment of cerebral global ischemia with Oct- $4^{+}$umbilical cord matrix cells. Stem Cells 25: 98-106.

Karahuseyinoglu S, Cinar O, Kilic E, et al. 2007; Biology of stem cells in human umbilical cord stroma: in situ and in vitro surveys. Stem Cells 25: 319-331.

Kasukawa Y, Miyakoshi N, Mohan S. 2004; The anabolic effects of GH/IGF system on bone. Curr Pharm Des 10: 2577-2592.

Kim HJ, Zhao H, Kitaura H, et al. 2006; Glucocorticoids suppress bone formation via the osteoclast. J Clin Invest 116: 2152-2160.

Koch H, Jadlowiec JA, Campbell PG. 2005; Insulin-like growth factor-I induces early osteoblast gene expression in human mesenchymal stem cells. Stem Cells Dev 14: 621-631.

Komori T, Yagi H, Nomura S, et al. 1997; Targeted disruption of Cbfa1 results in a complete lack of bone formation owing to maturational arrest of osteoblasts. Cell 89: 755-764.

Landis WJ. 1999; An overview of vertebrate mineralization with emphasis on collagen-mineral interaction. Gravit Space Biol Bull 12: $15-26$.

Lee SY, Miwa M, Sakai Y, et al. 2007; In vitro multipotentiality and characterization of human unfractured traumatic hemarthrosisderived progenitor cells: a potential cell source for tissue repair. $J$ Cell Physiol 210: 561-566.

Linkhart TA, Mohan S, Baylink DJ. 1996; Growth factors for bone growth and repair: IGF, TGF $\beta$ and BMP. Bone 19: S 1-12.

Livak KJ, Schmittgen TD. 2001; Analysis of relative gene expression data using real-time quantitative PCR and the $2[-\Delta \Delta \mathrm{C}(\mathrm{T})]$ method. Methods 25: 402-408.

Lund RD, Wang S, Lu B, et al. 2007; Cells isolated from umbilical cord tissue rescue photoreceptors and visual functions in a rodent model of retinal disease. Stem Cells 25: 602-611.

Lu LL, Liu YJ, Yang SG, et al. 2006; Isolation and characterization of human umbilical cord mesenchymal stem cells with haematopoiesis-supportive function and other potentials. Haematologica 91: 1017-1026.
Marcacci M, Kon E, Moukhachev V, et al. 2007; Stem cells associated with macroporous bioceramics for long bone repair: 6- to 7-year outcome of a pilot clinical study. Tissue Eng 13: 947-955.

Martin JA, Hamilton BE, Sutton PD, et al. 2007; Births: final data for 2005. Natl Vital Statist Rep 56: 1.

Meinel L, Karageorgiou V, Fajardo R, et al. 2004; Bone tissue engineering using human mesenchymal stem cells: effects of scaffold material and medium flow. Ann Biomed Eng 32: 112-122.

Mueller SM, Glowacki J. 2001; Age-related decline in the osteogenic potential of human bone marrow cells cultured in threedimensional collagen sponges. J Cell Biochem 82: 583-590.

Murata H, Tanaka H, Taguchi T, et al. 2004; Dexamethasone induces human spinal ligament derived cells toward osteogenic differentiation. $J$ Cell Biochem 92: 715-722.

Partridge NC, Frampton RJ, Eisman JA, et al. 1980; Receptors for $1,25(\mathrm{OH})_{2}$-vitamin D3 enriched in cloned osteoblast-like rat osteogenic sarcoma cells. FEBS Lett 115: 139-142.

Pittenger MF, Mackay AM, Beck SC, et al. 1999; Multilineage potential of adult human mesenchymal stem cells. Science 284: 143-147.

Quarto R, Mastrogiacomo M, Cancedda R, et al. 2001; Repair of large bone defects with the use of autologous bone marrow stromal cells. N Engl J Med 344: 385-386.

Ross RJ, Rodriguez-Arnao J, Bentham J, Coakley JH. 1993; The role of insulin, growth hormone and IGF-I as anabolic agents in the critically ill. Intens Care Med 19(suppl 2): S54-57.

Sarugaser R, Lickorish D, Baksh D, et al. 2005; Human umbilical cord perivascular (HUCPV) cells: a source of mesenchymal progenitors. Stem Cells 23: 220-229.

Schroeder TM, Jensen ED, Westendorf JJ. 2005; Runx2: a master organizer of gene transcription in developing and maturing osteoblasts. Birth Defects Res C Embryol Today 75: 213-225.

Sumanasinghe RD, Osborne JA, Loboa EG. 2008; Mesenchymal stem cell-seeded collagen matrices for bone repair: effects of cyclic tensile strain, cell density, and media conditions on matrix contraction in vitro. J Biomed Mater Res A 88: 778-786.

ter Brugge PJ, Jansen JA. 2002; In vitro osteogenic differentiation of rat bone marrow cells subcultured with and without dexamethasone. Tissue Eng 8: 321-331.

Tou L, Quibria N, Alexander JM. 2003; Transcriptional regulation of the human Runx2/Cbfa1 gene promoter by bone morphogenetic protein-7. Mol Cell Endocrinol 205: 121-129.

Troyer DL, Weiss ML. 2008; Wharton's jelly-derived cells are a primitive stromal cell population. Stem Cells 26: 591-599.

Walsh S, Jordan GR, Jefferiss C, et al. 2001; High concentrations of dexamethasone suppress the proliferation but not the differentiation or further maturation of human osteoblast precursors in vitro: relevance to glucocorticoid-induced osteoporosis. Rheumatology (Oxf) 40: 74-83.

Wang HS, Hung SC, Peng ST, et al. 2004; Mesenchymal stem cells in the Wharton's jelly of the human umbilical cord. Stem Cells 22: 1330-1337.

Wang L, Lazebnik M, Detamore MS. 2009; Hyaline cartilage cells outperform mandibular condylar cartilage cells in a TMJ fibrocartilage tissue engineering application. Osteoarthr Cartilage 17(3): 346-353.

Wang L, Seshareddy K, Weiss ML, Detamore MS. 2008; Effect of initial seeding density on human umbilical cord mesenchymal stromal cells for fibrocartilage tissue engineering. Tissue Eng A 1-9 [Epub ahead of print].

Wu KH, Zhou B, Lu SH, et al. 2007; In vitro and in vivo differentiation of human umbilical cord derived stem cells into endothelial cells. $J$ Cell Biochem 100: 608-616.

Yoshimoto H, Shin YM, Terai H, Vacanti JP. 2003; A biodegradable nanofiber scaffold by electrospinning and its potential for bone tissue engineering. Biomaterials 24: 2077-2082.

Yuan X, Arthur FTM, Yao K. 2002; In vitro degradation of poly(Llactic acid) fibers in phosphate buffered saline. J Appl Polym Sci 85: 936-943.

Zhang ZY, Teoh SH, Chong MS, et al. 2008; Superior osteogenic capacity for bone tissue engineering of fetal compared to perinatal and adult mesenchymal stem cells. Stem Cells 1-5 [Epub ahead of print]. 